\title{
Dietary determinants of micronutrient intake in older Irish adults
}

\author{
L. Kehoe ${ }^{1}$, J. Walton ${ }^{1}$, B. McNulty ${ }^{2}$, A. Nugent ${ }^{2}$ and A. Flynn ${ }^{1}$ \\ ${ }^{1}$ School of Food and Nutritional Sciences, University College Cork, Republic of Ireland and ${ }^{2}$ UCD Institute of Food \\ and Health, University College Dublin, Belfield, Dublin 4, Republic of Ireland
}

It is widely recognised that older adults are at greater risk of nutritional deficiencies due to the normal physiological changes that occur with ageing. It has previously been reported that older Irish adults have inadequate intakes of vitamin $\mathrm{C}$, vitamin $\mathrm{D}$, folate, calcium and magnesium. ${ }^{(1)}$ The objective of this analysis was to identify the dietary determinants of micronutrient intakes in older Irish adults ( $\geqslant 65$ years) based on data from the National Adult Nutrition Survey (2008-2010) (www.iuna.net). A 4-day food record was used to collect food intake data of 226 older Irish adults. Analysis of dietary intake data was carried out using WISP ${ }^{\complement}$ based on McCance and Widdowson's The Composition of Foods ${ }^{(2)}$ and the Irish Food Composition Database. ${ }^{(3)}$ On the basis of mean daily intake for each micronutrient, participants were divided into three intake groups: low medium or high consumers, stratified by gender. The food groups which accounted for the greatest proportion of the difference in intakes between the high (top third) and low (bottom third) consumers of each micronutrient were identified.

\begin{tabular}{|c|c|c|c|c|}
\hline \multirow[b]{2}{*}{ Nutrient } & \multicolumn{3}{|c|}{ Mean daily intake } & \multirow[b]{2}{*}{$\%$ Contribution of food groups to the difference between high and low consumers } \\
\hline & Low & High & Difference & \\
\hline Vitamin $\mathrm{C}(\mathrm{mg})$ & 32 & 220 & 188 & $\begin{array}{l}\text { Nutritional supplements (50), Fruit \& fruit juices (33), Vegetables \& veg dishes (8), Potatoes \& potato dishes } \\
\text { (2) }\end{array}$ \\
\hline Vitamin D $(\mu \mathrm{g})$ & $2 \cdot 1$ & $15 \cdot 2$ & $13 \cdot 1$ & Nutritional supplements (57), Fish \& fish dishes (22), Milks (7), Fat spreads (7) \\
\hline $\begin{array}{l}\text { Dietary Folate Equivalents } \\
(\mu \mathrm{g})\end{array}$ & 183 & 954 & 771 & Nutritional supplements (39), Fat spreads (21), Milks (16), RTEBC (8) \\
\hline Calcium (mg) & 525 & 1501 & 977 & Nutritional supplements (33), Milks (30), Cheeses (9), Bread \& rolls (7), Yoghurts (5) \\
\hline Magnesium (mg) & 170 & 390 & 220 & Bread \& rolls (17), Nutritional supplements (13), RTEBC (12), Milks (10), Beverages (10) \\
\hline
\end{tabular}

RTEBC $=$ Ready-to-eat breakfast cereals

Nutritional supplements were the key contributors to the difference in intakes between high and low consumers of vitamin C, vitamin D, dietary folate equivalents (DFE) and calcium. In relation to the food groups which contributed to the difference in intakes between consumer groups, fruit \& fruit dishes were important for vitamin C, fish \& fish dishes for vitamin D and fat spreads for DFE. For calcium, milks were also a signifigant contributor to the intake difference between high and low consumers. The key contributors to the difference in intakes between high and low magnesium consumers were bread \& rolls, nutritional supplements and ready-to-eat breakfast cereals. Other food groups were important sources of individual micronutrients but did not contribute to the difference in intakes between high and low consumers (e.g. the contribution of meat \& meat products to magnesium \& vitamin $\mathrm{D}$ intake and breads \& rolls to DFE intake). These findings will aid in the development of dietary strategies to improve micronutrient intakes in older adults.

The project was funded by the Irish Department of Agriculture, Food and the Marine under the Food Institutional Research Measure (FIRM)

1. Hennessy Á, Walton J, McNulty B et al. (2014) Micronutrient intakes and adequacy of intake in older adults in Ireland. Proceedings of the Nutrition Society $\mathbf{7 3}$, null-null.

2. Food Standards Agency (2002b) McCance \& Widdowson's The Composition of Foods Fifth \& Sixth Editions including supplemental volumes. Cambridge: Royal Society of Chemistry.

3. Black LJ, Ireland J, Møller A et al. (2011) Development of an on-line Irish food composition database for nutrients. J Food Compost Anal 24, $1017-1023$. 